\title{
Thermodynamic signature of growing amorphous order in glass-forming liquids
}

\author{
G. BIROLI' ${ }^{1}$, J.-P. BOUCHAUD², A. CAVAGNA ${ }^{3}$, T. S. GRIGERA4,5* AND P. VERROCCHIO ${ }^{6}$ \\ ${ }^{1}$ CEA, DSM, Institut de Physique Théorique, IPhT, CNRS, MPPU, URA2306, Saclay, F-91191 Gif-sur-Yvette, France \\ ${ }^{2}$ Science \& Finance, Capital Fund Management, 6 Bd Haussmann, 75009 Paris, France \\ ${ }^{3}$ Centre for Statistical Mechanics and Complexity (SMC), CNR-INFM, Via dei Taurini 19, 00185 Roma, Italy \\ ${ }^{4}$ Instituto de Investigaciones Fisicoquímicas Teóricas y Aplicadas (INIFTA - CCT La Plata) and Departamento de Física, Facultad de Ciencias Exactas, \\ Universidad Nacional de La Plata, c.c. 16, suc. 4, 1900 La Plata, Argentina \\ ${ }^{5}$ Consejo Nacional de Investigaciones Científicas y Técnicas, c.c. 16, suc. 4, 1900 La Plata, Argentina \\ ${ }^{6}$ Dipartimento di Fisica, Università di Trento, via Sommarive 14, 38050 Povo, Trento, Italy \\ *e-mail: tgrigera@inifta.unlp.edu.ar
}

Supercooled liquids exhibit a pronounced slowdown of their dynamics on cooling ${ }^{1}$ without showing any obvious structural or thermodynamic changes ${ }^{2}$. Several theories relate this slowdown to increasing spatial correlations $s^{3-6}$. However, no sign of this is seen in standard static correlation functions, despite indirect evidence from considering specific heat ${ }^{7}$ and linear dielectric susceptibility $^{8}$. Whereas the dynamic correlation function progressively becomes more non-exponential as the temperature is reduced, so far no similar signature has been found in static correlations that can distinguish qualitatively between a high-temperature and a deeply supercooled glass-forming liquid in equilibrium. Here, we show evidence of a qualitative thermodynamic signature that differentiates between the two. We show by numerical simulations with fixed boundary conditions that the influence of the boundary propagates into the bulk over increasing length scales on cooling. With the increase of this static correlation length, the influence of the boundary decays non-exponentially. Such long-range susceptibility to boundary conditions is expected within the random first-order theory ${ }^{4,9,10}$ (RFOT) of the glass transition. However, a quantitative account of our numerical results requires a generalization of RFOT, taking into account surface tension fluctuations between states.

Inspired by critical phenomena, it is natural to expect that the slowing down of the dynamics is related to the vicinity of a thermodynamic phase transition, where some kind of long-range order would set $\mathrm{in}^{11}$. This is the spirit of different recent theories ${ }^{4,9,12-14}$, but seems at odds with others ${ }^{5,15}$, at least at first sight. In particular, the crucial physical mechanism at the root of random first-order theory ${ }^{4}$ (RFOT) is the emergence of long-range amorphous order, the precise definition and quantitative characterization of which is however far from obvious. Dynamic heterogeneities ${ }^{16}$ do show a growing dynamic correlation length accompanying the glass transition, both experimentally ${ }^{17}$ and numerically ${ }^{18}$. This is certainly a first important step, but not sufficient to prune down-even at a qualitative level-different theories of the glass transition. In particular, it is not clear whether this phenomenon is due to an underlying static or purely dynamic phase transition.

The approach followed here is based on the very definition of a thermodynamic phase transition, where the effect of boundary conditions becomes long-ranged. The problem is that for glasses there are no natural boundary conditions, because these should be as 'random' as the bulk amorphous states that they favour. A possible solution is to use equilibrium liquid configurations to define the boundary ${ }^{19}$. In the context of RFOT, this was suggested in ref. 9 (and further discussed in ref. 11), but the scope and some conclusions of this Gedankenexperiment are more general ${ }^{19-21}$. Starting from a given equilibrium configuration, we freeze the motion of all particles outside a cavity of radius $R$ and study the thermodynamics of the mobile particles with boundary conditions imposed by the frozen ones. Defining a suitable overlap (inside the cavity) between the original equilibrated configuration and the configurations equilibrated with the boundary pinning field, the existence of 'order' on a scale $\xi$ implies a large overlap (or 'point-to-set' correlation) for $R \ll \xi$ and a small one for $R \gg \xi$.

Such correlation length arises in RFOT $^{9}$, if we assume (or find by approximate calculations ${ }^{10,22}$ ) that there are many amorphous metastable states, $\mathcal{N} \simeq \exp \left[R^{d} \Sigma\right]$ inside the cavity $(\Sigma(T)$ is the configurational entropy, or complexity). The particles in the cavity can be either in the original state $\alpha$ in which the outside particles have been frozen or in any of the other states. The probability to flip to a different state $\gamma$ is determined by the balance between the free-energy loss due to mismatch between $\alpha$ and $\gamma, \Upsilon(T) R^{\theta}$, and the gain from the entropic term $T R^{d} \Sigma(T)(\Upsilon$ is a generalized surface tension and $\theta \leq d-1)$. A crossover between a boundary-dominated regime (large overlap) and an entropy-dominated regime (small overlap) is expected for $R=\xi \propto(\Upsilon / T \Sigma)^{(1 / d-\theta)}$. Note that this length diverges at the Kauzmann temperature $T_{\mathrm{K}}$ where $\Sigma\left(T_{\mathrm{K}}^{+}\right) \rightarrow 0$.

This Gedankenexperiment was realized numerically by three of us in ref. 23. This first study indeed suggested a growing static length, but this length was very small, and no sharp transition between high and low overlap was observed, in contrast with expectations based on RFOT. Is RFOT possibly in contradiction with numerical experiments? What is the physical meaning of the increasingly long-range susceptibility to boundary conditions if it is characterized by such a small length? To answer these crucial questions, we have carried out new simulations that measure the local overlap at the centre of the cavity. In fact, a major difficulty in interpreting the results of ref. 23 is that the overlap was computed 


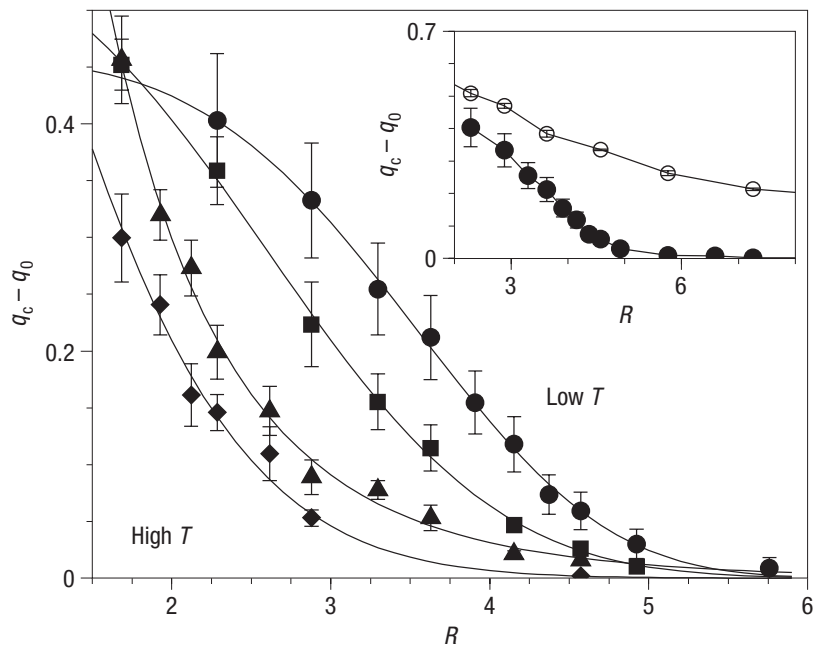

Figure 1 Change of the overlap with mobile cavity size. Overlap at the centre of the mobile cavity versus radius $R$ of the cavity, for temperatures $T=0.482$ (diamonds), 0.350 (triangles), 0.246 (squares) and 0.203 (circles). Lines are fits to equation (1). Inset: Comparison of $q_{\mathrm{c}}(R)-q_{0}$ at $T=0.203$ (filled circles) with the overlap $Q(R)-q_{0}$ integrated over the whole sphere (open circles, data ref. 23). The local observable $q_{c}(R)$ shows a much sharper behaviour. Error bars were obtained from a jack-knife estimate from sample-to-sample fluctuations.

as an average over the whole cavity. As a result, the overlap drop due to the (putative) change of state of the cavity is mingled with the decay of the overlap expected from a trivial weakening of the surface pinning field for larger spheres. This effect is indeed present even in the single-state case $\mathrm{e}^{21}$ at all temperatures. As we shall show, analysing the behaviour of the local overlap at the centre of the cavity yields sharper results that allow us to answer, at least partially, the above questions.

We study a soft-sphere model ${ }^{24}$ that we can equilibrate below the mode-coupling transition temperature $T_{\mathrm{MC}}=0.226$ (ref. 25) and for large systems (see the Methods section). After equilibration, several independent reference configurations are chosen as starting points for runs with all but $M$ particles frozen. These mobile particles are confined inside a sphere of radius $R$ such that the density inside equals the bulk density. After the confined runs reach equilibrium, the local overlap at the centre $q_{c}(R)$ is measured. To define $q_{\mathrm{c}}(R)$, we partition the simulation box into many small cubic boxes of side $\ell$, such that the probability of finding more than one particle in a single box is negligible. Let $n_{i}$ equal the number of particles in box $i$, then

$$
q_{c}(R)=\frac{1}{\ell^{3} N_{i}} \sum_{i \in v}\left\langle n_{i}\left(t_{0}\right) n_{i}\left(t_{0}+\infty\right)\right\rangle,
$$

where the sum runs over all boxes within a small volume $v$ at the centre of the sphere, $N_{i}$ is the number of boxes and $\langle\cdots\rangle$ means thermal average. To minimize statistical uncertainty without losing the local nature of $q_{\mathrm{c}}(R)$, we choose $N_{i}=v / \ell^{3}=125$. Normalization is such that the overlap of two identical configurations is 1 on average, whereas for totally uncorrelated configurations $q_{\mathrm{c}}=q_{0} \equiv \ell^{3}=0.062876$.

We show $q_{c}(R)-q_{0}$ for several temperatures in Fig. 1 . The decay becomes slower at lower temperature: the effect of boundary conditions propagates on larger length scales. This clearly shows the growth of static order that expands into the bulk in the deeply supercooled phase. The most striking result, however, is that at low
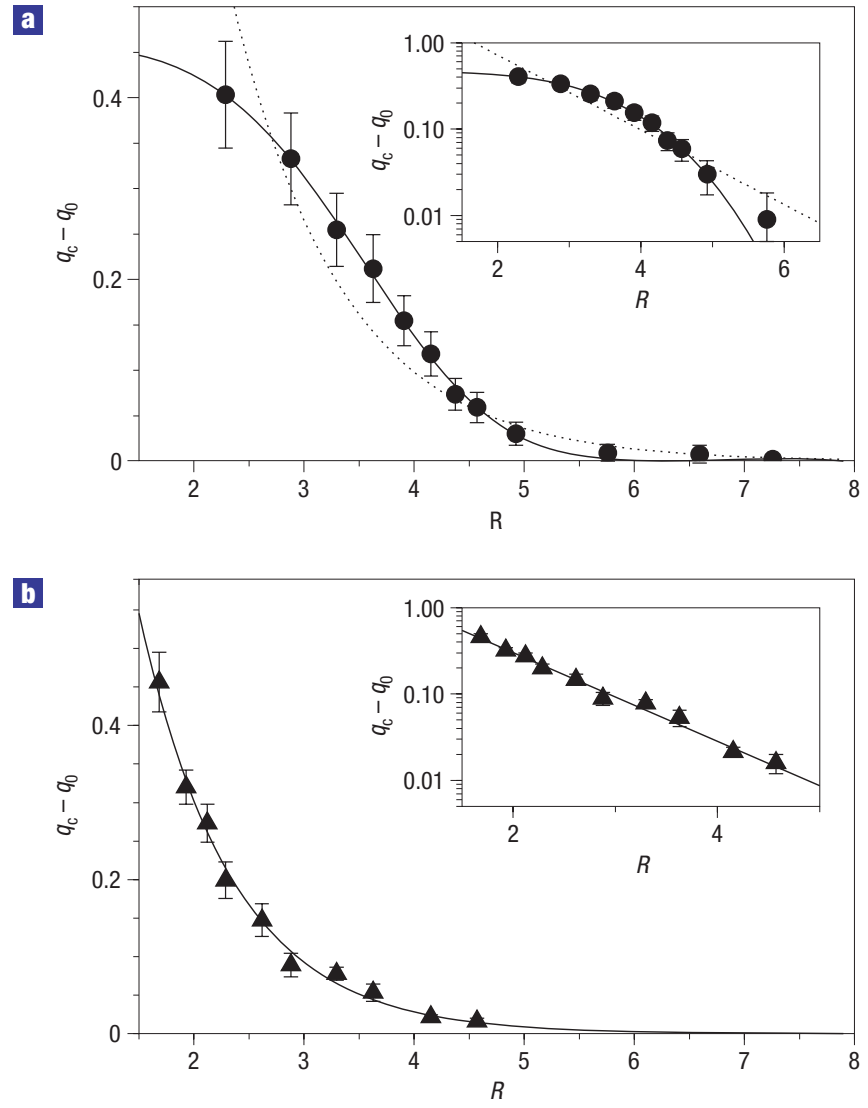

Figure 2 Non-exponentiality of the overlap decay at low temperatures. a, Local overlap at the centre of the mobile cavity versus $R$ for $T=0.203$ (circles) with fits to a compressed exponential (solid line) and a simple exponential (dotted line). The best exponential fit is rather poor. Inset: Same data in semilogarithmic axes, where a simple exponential looks like a straight line. $\mathbf{b}$, As in a but for $T=0.350$. The exponential fit (line) is good at this temperature, as can be seen in the inset, which shows the same data in semilogarithmic axes. Error bars as before.

temperatures the decay is no longer the simple exponential that prevails in a standard liquid state ${ }^{21}$. The relaxation can be fitted by a 'compressed exponential',

$$
q_{\mathrm{c}}(R)-q_{0}=\Omega \exp \left[-(R / \xi)^{\zeta}\right], \quad \zeta \geq 1
$$

where $\zeta$ increases at low temperatures (Fig. 2a) above its hightemperature liquid value $\zeta=1$ (Fig. 2b). Larger values of $\zeta$ mean a sharper crossover between large and small overlaps. The best fit parameters are given in Table 1. Interestingly, the value of the length scale $\xi$ found here is significantly larger than those in ref. 23. This is partly due to the fact that $\zeta>1$ at low temperatures (see Supplementary Information for further discussion). The overlap $q_{\mathrm{c}}(R)$ therefore appears as a thermodynamic quantity able to single out the deeply supercooled liquid in terms of (1) a large correlation length $\xi$ and (2) an anomalous non-exponential relaxation, characterized by the exponent $\zeta>1$.

The growth of $\xi$ strongly suggests that the liquid state should be thought of as a mosaic of regions (transiently) ordered on a length scale $\xi$. Furthermore, following suggestions based on RFOT $^{4,9,12}$, it is natural to conjecture that we are probing the growth of positional amorphous order. Interestingly, in this scenario, all finite-point static correlations remain featureless, whereas the point-to-set 
Table 1 Best fit parameters as a function of temperature. Errors are jack-knife estimates.

\begin{tabular}{llll}
\hline$T$ & $\zeta$ & $\xi$ & $\Omega$ \\
\hline 0.482 & $1^{*}$ & $0.617(40)$ & $5.3(1.3)$ \\
0.350 & $1^{*}$ & $0.845(28)$ & $3.22(32)$ \\
0.246 & $2.79(52)$ & $3.04(24)$ & $0.552(61)$ \\
0.203 & $4.00(60)$ & $3.82(12)$ & $0.46(11)$ \\
\hline
\end{tabular}

*For the highest temperatures, the results quoted are for a simple exponential fit, which gives a very good description of the data with only two parameters. If nonetheless those data are fitted with the compressed exponential form, an exponent $\zeta$ compatible with 1 is obtained, albeit with very large $(60-80 \%)$ error.

correlations $^{11,26}$, captured by $q_{\mathrm{c}}(R)$, grow. The simplest version of RFOT predicts at low temperature a sharp crossover of $q_{\mathrm{c}}(R)$ for $R=\xi$ (ref. 27). Our numerical results show that this is not what happens: the crossover is much smoother than expected (see also the recent work ${ }^{28}$ on a $1-d$ Kac model).

To account for our numerical results, we now propose a natural generalization of RFOT, based on the idea that the effective interface tension, $\Upsilon$, is in fact state dependent. Repeating the argument of ref. 9 with this extra assumption, the probability that the cavity is found in a state $\gamma$ different from the outside pinning (frozen) state $\alpha$, is:

$$
\begin{aligned}
& p_{\text {out }}^{(\alpha)}(R)=\frac{\int \mathrm{d} \Upsilon \mathrm{e}^{R^{d} \Sigma^{*}-\beta \Upsilon R^{\theta}} p_{\alpha}\left(\Upsilon \mid f^{*}\right)}{1+\int \mathrm{d} \Upsilon \mathrm{e}^{R^{d} \Sigma^{*}-\beta \Upsilon R^{\theta}} p_{\alpha}\left(\Upsilon \mid f^{*}\right)}, \\
& p_{\text {in }}^{(\alpha)}(R)=1-p_{\text {out }}^{(\alpha)}(R),
\end{aligned}
$$

where $\beta=1 / T, f^{*}$ and $\Sigma^{*}=\Sigma\left(f^{*}\right)$ are respectively the free energy and configurational entropy of the equilibrium states and $p_{\alpha}(\Upsilon \mid f)$ is the distribution of surface tension for state $\alpha$ with other states of free energy $f$ (see the Methods section). As our simulations average over many realizations of the external state $\alpha$, we in fact compute $p_{\text {out }}^{(\alpha)}(R)$ averaged over all possible pinning states:

$$
\begin{aligned}
p_{\text {out }}(R) & =\sum_{\alpha} w_{\alpha} p_{\text {out }}^{(\alpha)}(R) \\
& =\sum_{\alpha} w_{\alpha} \frac{\int \mathrm{d} \Upsilon \mathrm{e}^{R^{d} \Sigma^{*}-\beta \Upsilon R^{\theta}} p_{\alpha}\left(\Upsilon \mid f^{*}\right)}{1+\int \mathrm{d} \Upsilon \mathrm{e}^{R^{d} \Sigma^{*}-\beta \Upsilon R^{\theta}} p_{\alpha}\left(\Upsilon \mid f^{*}\right)},
\end{aligned}
$$

where $w_{\alpha}$ is the thermodynamic weight of each state. The leading contribution of the integral over $\Upsilon$ is shown in the Methods section to be $\mathrm{e}^{-\beta y_{\alpha} R^{\theta}}$, where $y_{\alpha}$ is the lower edge of $p_{\alpha}(\Upsilon \mid f)$ and where the exponent $\theta$ is possibly renormalized (this might explain why the effective value of $\theta$ can be less than the naively expected value $d-1)$. Introducing $P(Y)=\sum_{\alpha} w_{\alpha} \delta\left(Y-y_{\alpha}\right)$, we find

$$
p_{\text {out }}(R)=\int \mathrm{d} Y P(Y) \frac{\mathrm{e}^{R^{d} \Sigma^{*}-\beta Y R^{\theta}}}{1+\mathrm{e}^{R^{d} \Sigma^{*}-\beta Y R^{\theta}}} .
$$

The simple mosaic result of ref. 9 is recovered setting $p_{\alpha}(\Upsilon \mid f)=\delta\left(\Upsilon-\Upsilon_{0}\right)$ and $P(Y)=\delta\left(Y-Y_{0}\right)$. Expression (4) can be simplified further by noticing that the approximation $\left(1+\mathrm{e}^{R^{d} \Sigma^{*}-\beta Y R^{\theta}}\right)^{-1} \approx \Theta\left(Y-T \Sigma^{*} R^{d-\theta}\right)$ holds even for rather small values of $R$ ( $\Theta$ is the step function). Hence,

$$
p_{\text {out }}(R)=\int_{0}^{T \Sigma^{*} R^{d-\theta}} P(Y) \mathrm{d} Y .
$$

The overlap at the centre of the cavity is given by $q_{\mathrm{c}}(R)=p_{\text {in }}(R) q_{1}+p_{\text {out }}(R) q_{0}$, where $q_{1}$ is the self-overlap of the cavity (which is not necessarily 1 at finite temperatures). Using equation (5), we finally obtain

$$
q_{\mathrm{c}}(R)-q_{0}=\left(q_{1}-q_{0}\right) \int_{T \Sigma^{*} R^{d-\theta}}^{\infty} \mathrm{d} Y P(Y) .
$$

At this point, some assumption about $P(Y)$ needs to be made. An interesting parameterization is

$$
\begin{aligned}
P(Y) & =\frac{v}{Y_{\mathrm{c}}}\left(\frac{Y}{Y_{\mathrm{c}}}\right)^{v-1} \exp \left[-\left(Y / Y_{\mathrm{c}}\right)^{\nu}\right] \\
& =-\frac{\mathrm{d}}{\mathrm{d} Y} \exp \left[-\left(Y / Y_{\mathrm{c}}\right)^{v}\right]
\end{aligned}
$$

$(v>0)$, which describes either a stiff distribution (small values of $Y$ suppressed) at large $v$ or a soft one (small values of $Y$ enhanced) for small $v . Y_{c}$ is the typical value of $\Upsilon$. The standard mosaic picture corresponds to $v \rightarrow \infty$. Equation (6) now reads, explicitly:

$$
q_{c}(R)-q_{0}=\left(q_{1}-q_{0}\right) \exp \left[-(R / \xi)^{v(d-\theta)}\right],
$$

where the usual RFOT relation between $\xi$ and $\Sigma$ is recovered, $\xi=\left(Y_{c} / T \Sigma^{*}\right)^{1 /(d-\theta)}$. As in the Adam-Gibbs treatment ${ }^{29}$, the growth of the length is caused by the decreasing of the configurational entropy at low temperature. The expression (7) is the compressed exponential form (1) used above to fit our numerical data. Within this framework, the thermodynamic anomaly $\zeta=v(d-\theta)$ is directly related (at low temperatures) to the exponent $v$ describing the surface tension distribution.

A non-trivial $P(Y)$ could arise for two main reasons. One is strong pre-asymptotic corrections to standard RFOT: although the surface tension between two typical states could be self-averaging in the $R \rightarrow \infty$ limit, fluctuations can be state dependent and important at finite $R$. This is often what happens for interfaces in random media (see the Supplementary Information). The second possibility is that the effective interface tension fluctuates even in the large- $R$ limit. If the exponent $\theta$ turns out to be less than $d-1$, as suggested by phenomenological arguments in ref. 4, then the interface should be a highly fluctuating object. We find that $\zeta$, and therefore $v$, increases when $T$ decreases, indicating that the crossover of $q_{\mathrm{c}}(R)$ becomes sharper at larger sizes (Fig. 1). This is compatible with a finite-size-effect scenario. This behaviour is also expected within the RFOT scenario that predicts a vanishing surface tension at the mode-coupling transition $T_{\mathrm{MC}}$, which behaves as a spinodal point. Coherent amorphous order droplets should therefore be fractal around $T_{\mathrm{MC}}$ and compact below $^{30}$, which suggests an increase of the effective value of $v$ as $T$ decreases. A first-principles RFOT computation of $q_{\mathrm{c}}(R)$ for the model we simulated would be very instrumental to clarify this issue.

We have developed a theoretical framework, based on a generalization of RFOT, that explains these results as a one-state to multistate transition governed by the surface tension distribution. The sharpening of the decay at low temperature corresponds to more and more regions developing a large surface tension. From a more general perspective, our numerical results strongly support a mosaic picture where the supercooled liquid is characterized by a 'hidden' static order on an increasingly larger scale on cooling. Although this is a natural consequence of RFOT, other theoretical approaches may also account for these phenomena at least on a qualitative level: in particular the frustration-limited domain theory ${ }^{14}$, the effective potential approach ${ }^{31}$ and, perhaps surprisingly, some kinetically constrained models (see ref. 20). The main difference is the physical origin of the growing static length 


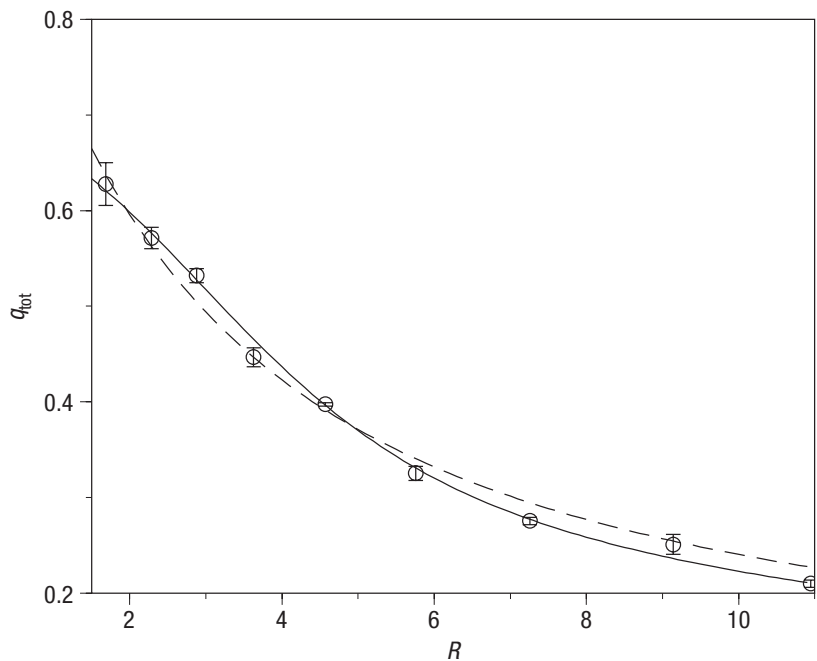

Figure 3 Comparison with previous numerical results. Total overlap of the mobile cavity at $T=0.203$ (data taken from ref. 23). The dashed line shows the fit with one-state theory, whereas the solid line shows the fit with the generalized RFOT prediction (8). Error bars as before.

scale: within RFOT and at variance with other approaches, it is closely linked to the decrease of the configurational entropy. Our work opens the way to a quantitative study of this issue and, hence, to a clear-cut test of RFOT as a valid theory of the glass transition. From a more theoretical point of view, several crucial questions remain elusive: can an RFOT-like transition exist outside mean-field? How precisely can amorphous metastable states be defined? Does the notion of effective surface tension between these states make sense? A definitive test of the mosaic scenario requires finding a way to measure directly this surface tension and its distribution in the deeply supercooled phase. Work in this direction is in progress.

\section{METHODS}

\section{SIMULATION}

We have studied the soft-sphere binary mixture ${ }^{24}$, a fragile model glass-former. In addition to capturing the essential features of fragile glasses, this model can be thermalized below the mode-coupling temperature with the swap Monte Carlo algorithm of ref. 32. Particles are of unit mass and belong to one of two species $\mu=1,2$, present in equal amounts and interacting through a potential

$$
V=\sum_{i>j}^{N} v_{i j}\left(\left|\mathbf{r}_{i}-\mathbf{r}_{j}\right|\right)=\sum_{i>j}^{N}\left[\frac{\sigma_{\mu(i)}+\sigma_{\mu(j)}}{\left|\mathbf{r}_{i}-\mathbf{r}_{j}\right|}\right]^{12},
$$

where the radii $\sigma_{\mu}$ are fixed by the conditions $\sigma_{2} / \sigma_{1}=1.2$, $\left(2 \sigma_{1}\right)^{3}+2\left(\sigma_{1}+\sigma_{2}\right)^{3}+\left(2 \sigma_{2}\right)^{3}=4 \ell_{0}^{3}$ and $\ell_{0}$ is the unit of length. The particle density is $\rho=N / V=l_{0}^{-3}$. A smooth long-range cutoff is imposed setting $v_{i j}(r)=B_{i j}(a-r)^{3}+C_{i j}$ for $r>r_{\mathrm{c}}=\sqrt{3}$ and $v_{i j}(r)=C_{i j}$ for $r>a$, where $a, B_{i j}$ and $C_{i j}$ are fixed by requiring continuity up to the second derivative of $v_{i j}(r)$. Temperature is measured in units of energy. To obtain the reference configurations, four to eight replicas of systems with $N=2,048$ or $N=16,384$ were equilibrated in a cubic box with periodic boundary conditions. Then the overlap was computed in systems with frozen boundaries and $M$ mobile particles, with $M=20,30,40,50,100,150,200,300,400,800,1,600,3,200$ mobile particles $(1.684 \leq R \leq 9.142)$. The results are averaged over 8 to 32 outer states (reference configurations). Data were collected for at least 10 relaxation times (up to $10^{6}$ Monte Carlo steps), after discarding an initial portion of at least one relaxation time.

\section{OVERLAP}

To obtain equation (2), write the partition function for the mobile cavity surrounded by pinning state $\alpha$,

$$
\mathcal{Z}_{\mathrm{c}}=\mathrm{e}^{-\beta R^{d} f_{\alpha}}+\sum_{\gamma \neq \alpha} \mathrm{e}^{-\beta R^{d} f_{\gamma}-\beta R^{\theta} \Upsilon_{\alpha \gamma}},
$$

so that

$$
p_{\text {out }}^{(\alpha)}(R)=\frac{\sum_{\gamma \neq \alpha} \mathrm{e}^{-\beta R^{d} f_{\gamma}-\beta R^{\theta} r_{\alpha \gamma}}}{\mathcal{Z}_{\mathrm{c}}} .
$$

Introducing $\mathcal{N}_{\alpha}(f, \Upsilon)=\sum_{\gamma} \delta\left(f-f_{\gamma}\right) \delta\left(\Upsilon-\Upsilon_{\alpha \gamma}\right)$, the sum can be written

$$
\begin{aligned}
\sum_{\gamma \neq \alpha} \mathrm{e}^{-\beta R^{d} f_{\beta}-\beta R^{\theta} \Upsilon_{\alpha \gamma}} & =\int \mathrm{d} f \int \mathrm{d} \Upsilon \mathrm{e}^{-\beta R^{d} f-\beta R^{\theta} \Upsilon} \mathcal{N}(f, \Upsilon), \\
& =\int \mathrm{d} f \int \mathrm{d} \Upsilon \mathrm{e}^{-\beta R^{d} f-\beta R^{\theta} \Upsilon+R^{d} \Sigma(f)} p_{\alpha}(\Upsilon \mid f),
\end{aligned}
$$

where in the last equality we have defined $p_{\alpha}(\Upsilon \mid f)=\mathcal{N}_{\alpha}(f, \Upsilon) / \mathcal{N}(f)$, and $\mathcal{N}(f)=\exp [\Sigma(f)]$ is the number of states with free energy $f$. Equation $(2)$ follows approximating the integral over $f$ with the saddle-point method, which picks $f=f^{*}$ as the dominant contribution. $p_{\alpha}(\Upsilon \mid f)$ is the fraction of states $\gamma$ (inside the cavity) with free energy $f$ and effective interface tension $\Upsilon$, which we assume to be $R$ independent (actually, some $R$ dependence would not affect the result; the important requirement is that this dependence does not lead to values exponentially large or small in $R$ ).

Now the integral in equations (2) and (3) can be simplified using the saddle-point method, which is a very good approximation even for rather small values of $R$. Because of the exponential term in $R^{\theta}$, the integral is dominated by the lowest values of $\Upsilon$ supported by the distribution $p_{\alpha}(\Upsilon \mid f)$. There are two possibles cases. (1) If this function has a left edge, that is, it vanishes for $\Upsilon<y_{\alpha}$, we find up to subleading terms: $\int \mathrm{d} \Upsilon p_{\alpha}(\Upsilon \mid f) \mathrm{e}^{-\beta \Upsilon R^{\theta}} \simeq \mathrm{e}^{-\beta y_{\alpha} R^{\theta}}$. Otherwise, (2) there are arbitrarily small effective tensions $\Upsilon$. Remarkably, in this case, depending on the form of $p_{\alpha}(\Upsilon \mid f)$ at small $\Upsilon$, we can obtain a renormalization of $\theta$. For instance, in the case $p_{\alpha}(\Upsilon \mid f) \simeq \exp \left(-c_{\alpha} / \Upsilon^{a}\right)$, where $a$ is a positive exponent, we find $\int \mathrm{d} \Upsilon p_{\alpha}(\Upsilon \mid f) \mathrm{e}^{-\beta \Upsilon R^{\theta}} \simeq \mathrm{e}^{-\beta y_{\alpha}^{\prime} R^{\theta^{\prime}}}$, where $y_{\alpha}^{\prime}$ is a constant dependent on the outside state $\alpha$ and temperature and $\theta^{\prime}=a \theta /(a+1)<\theta$.

\section{COMPARISON WITH THE RESULTS OF REF. 23}

Following the same numeric protocol of this work, in ref. 23 the influence of the boundary conditions on the total overlap within the sphere was studied. It was observed that the decay of $q_{\text {tot }}(R)$ is described sufficiently well within the single-state framework and the standard RFOT scenario was ruled out. In this work, we show however that at low enough temperatures, neither the standard RFOT nor the single-state scenario accounts for the local overlap data and a generalized RFOT theory is presented that is far more successful.

We might retrospectively wonder if the data in ref. 23 could have been used to discriminate between the one-state and the generalized RFOT scenarios. The answer is no. Allowing for an $R$ dependence of $q_{0}$ and $q_{1}$ of the type suggested in ref. 23,

$$
q_{0 ; 1}(R)=3\left(1-q_{0 ; 1}^{*}\right)\left[\frac{1}{x}-\frac{2}{x^{2}}+\frac{2\left(1-\mathrm{e}^{-x}\right)}{x^{3}}\right]+q_{0 ; 1}^{*},
$$

with $x \equiv R / \lambda_{0 ; 1}$, the generalized RFOT prediction for $q_{\text {tot }}(R)$ reads now:

$$
q_{\text {tot }}(R)=q_{0}(R)+\left(q_{1}(R)-q_{0}(R)\right) \exp \left[-(R / \xi)^{\zeta}\right] .
$$

Figure 3 shows that the generalized RFOT encoded in (8) and the one-state prediction given by the formula (6) of ref. 23 fit the total overlap data at $T=0.203$ at a comparable level of accuracy. Following Occam's razor principle ('entia non sunt multiplicanda praeter necessitatem'), the one-state theory should be chosen, which is the one with the smallest number of parameters.

\section{Received 31 March 2008; accepted 3 July 2008; published 10 August 2008.}

\section{References}

1. Ediger, M. D., Angell, C. A. \& Nagel, S. R. Supercooled liquids and glasses. J. Phys. Chem. 100 13200 (1996)

2. Leheny, R. L. et al. Structural studies of an organic liquid through the glass transition. J. Chem. Phys. 105, 7783-7794 (1996) 
3. Gibbs, J. H. \& DiMarzio, E. A. Nature of the glass transition and the glassy state. J. Chem. Phys. 28, 373-383 (1958)

4. Kirkpatrick, T. R., Thirumalai, D. \& Wolynes, P. G. Scaling concepts for the dynamics of viscous liquids near an ideal glassy state. Phys. Rev. A 40, 1045-1054 (1989).

5. Garrahan, J. P. \& Chandler, D. Geometrical explanation and scaling of dynamical heterogeneities in glass forming systems. Phys. Rev. Lett. 89, 035704 (2002).

6. Tarjus, G., Kivelson, S. A., Nussinov, Z. \& Viot, P. The frustration-based approach of supercooled liquids and the glass transition: a review and critical assessment. J. Phys. Condens. Matter 17, R1143 (2005)

7. Fernández, L. A., Martín-Mayor, V. \& Verrocchio, P. Critical behavior of the specific heat in glass formers. Phys. Rev. E 73, 020501 (2006).

8. Menon, N. \& Nagel, S. R. Evidence for a divergent susceptibility at the glass transition. Phys. Rev. Lett. 74, 1230-1233 (1995).

9. Bouchaud, J.-P. \& Biroli, G. On the Adam-Gibbs-Kirkpatrick-Thirumalai-Wolynes scenario for the viscosity increase in glasses. J. Chem. Phys. 121, 7347-7354 (2004).

10. Dzero, M., Schmalian, J. \& Wolynes, P. G. Activated events in glasses: The structure of entropic droplets. Phys. Rev. B 72, 100201 (2005).

11. Montanari, A. \& Semerjian, G. Rigorous inequalities between length and timescales in glassy systems. J. Stat. Phys. 125, 23-54 (2006).

12. Bouchaud, J.-P. \& Biroli, G. Nonlinear susceptibility in glassy systems: A probe for cooperative dynamical length scales. Phys. Rev. B 72, 064204 (2005).

13. Coluzzi, B., Mezard, M., Parisi, G. \& Verrocchio, P. Thermodynamics of binary mixture glasses. J. Chem. Phys. 111, 9039-9052 (1999).

14. Kivelson, D., Tarjus, G. \& Kivelson, S. A. A viewpoint, model and theory for supercooled liquids. Prog. Theor. Phys. Suppl. 126, 289-299 (1997).

15. Toninelli, C., Biroli, G. \& Fisher, D. S. Jamming percolation and glass transitions in lattice models. Phys. Rev. Lett. 96, 035702 (2006).

16. Ediger, M. D. Spatially heterogeneous dynamics in supercooled liquids. Annu. Rev. Phys. Chem. $\mathbf{5 1}$ 99-128 (2000).

17. Berthier, L. et al. Direct experimental evidence of a growing length scale accompanying the glass transition. Science 310, 1797-1800 (2005)

18. Toninelli, C., Wyart, M., Berthier, L., Biroli, G. \& Bouchaud, J.-P. Dynamical susceptibility of glass formers: Contrasting the predictions of theoretical scenarios. Phys. Rev. E 71, 041505 (2005).

19. Scheidler, P., Kob, W. \& Binder, K. The relaxation dynamics of a supercooled liquid confined by rough walls. J. Phys. Chem. B 108, 6673-6686 (2004).

20. Jack, R. L. \& Garrahan, J. P. Caging and mosaic length scales in plaquette spin models of glasses. J. Chem. Phys. 123, 164508 (2005).
21. Cammarota, C. \& Cavagna, A. A novel method for evaluating the critical nucleus and the surface tension in systems with first order phase transition. J. Chem. Phys. 127, 214703 (2007).

22. Franz, S. First steps of a nucleation theory in disordered systems. J. Stat. Mech. 2005, P04001 (2005).

23. Cavagna, A., Grigera, T. S. \& Verrocchio, P. Mosaic multistate scenario versus one-state description of supercooled liquids. Phys. Rev. Lett. 98, 187801 (2007).

24. Bernu, B., Hansen, J. P., Hiwatari, Y. \& Pastore, G. Soft-sphere model for the glass transition in binary alloys: Pair structure and self-diffusion. Phys. Rev. A 36, 4891-4903 (1987).

25. Roux, J.-N., Barrat, J.-L. \& Hansen, J.-P. Dynamical diagnostics for the glass transition in soft-sphere alloys. J. Phys. Condens. Matter 1, 7171-7186 (1989).

26. Mézard, M. \& Montanari, A. Reconstruction on trees and spin glass transition. J. Stat. Phys. 124, $1317-1350$ (2006).

27. Franz, S. \& Montanari, A. Analytic determination of dynamical and mosaic length scales in a Kac glass model. J. Phys. A 40, F251-F257 (2007).

28. Franz, S., Parisi, G. \& Ricci-Tersenghi, F. Mosaic length and finite interaction-range effects in a one dimensional random energy model. Preprint at $<$ http://arxiv.org/abs/0711.4780v2 > (2007).

29. Adam, G. \& Gibbs, J. H. On the temperature dependence of cooperative relaxation properties in glass-forming liquids. J. Chem. Phys. 43, 139-146 (1965).

30. Stevenson, J. D., Schmalian, J. \& Wolynes, P. G. The shapes of cooperatively rearranging regions in glass-forming liquids. Nature Phys. 2, 268-274 (2006).

31. Moore, M. A. \& Yeo, J. Thermodynamic glass transition in finite dimensions. Phys. Rev. Lett. 96 , 095701 (2006).

32. Grigera, T. S. \& Parisi, G. Fast Monte Carlo algorithm for supercooled soft spheres. Phys. Rev. E 63, $045102(2001)$.

Supplementary Information accompanies the paper at www.nature.com/naturephysics.

\section{Acknowledgements}

We thank C. Cammarota, L. A. Fernandez, G. Gradenigo, I. Giardina, A. Lefevre, V. Martín-Mayor, A. Montanari, G. Parisi, D. Reichman, M. Tarzia and F. Zamponi for useful discussions. G.B. and J.-P.B. are supported by ANR Grant DYNHET. T.S.G. thanks ECT* and Dipartimento di Fisica, Universitá di Trento for hospitality and partial support and acknowledges partial support from CONICET and ANPCyT (Argentina) and ICTP (Trieste, Italy).

\section{Author information}

Reprints and permission information is available online at http://npg.nature.com/reprintsandpermissions. Correspondence and requests for materials should be addressed to T.S.G. 